\title{
Towards an Assessment Framework for Congestion Management Mechanisms in Distribution Networks
}

Chen, Zhe; Ziras, Charalampos; Bindner, Henrik W.

Published in:

Proceedings of IEEE PES Innovation Smart Grid Technologies Europe 2021

Link to article, DOI:

10.1109/ISGTEurope52324.2021.9640095

Publication date:

2021

Document Version

Peer reviewed version

Link back to DTU Orbit

Citation (APA):

Chen, Z., Ziras, C., \& Bindner, H. W. (2021). Towards an Assessment Framework for Congestion Management Mechanisms in Distribution Networks. In Proceedings of IEEE PES Innovation Smart Grid Technologies Europe 2021 [319] IEEE. https://doi.org/10.1109/ISGTEurope52324.2021.9640095

\section{General rights}

Copyright and moral rights for the publications made accessible in the public portal are retained by the authors and/or other copyright owners and it is a condition of accessing publications that users recognise and abide by the legal requirements associated with these rights.

- Users may download and print one copy of any publication from the public portal for the purpose of private study or research.

- You may not further distribute the material or use it for any profit-making activity or commercial gain

- You may freely distribute the URL identifying the publication in the public portal 


\title{
Towards an Assessment Framework for Congestion Management Mechanisms in Distribution Networks
}

\author{
Zhe Chen, Charalampos Ziras and Henrik W. Bindner \\ Department of Electrical Engineering \\ Technical University of Denmark \\ Copenhagen, Denmark \\ \{zhech, chazi, hwbi\}@elektro.dtu.dk
}

\begin{abstract}
The increasing penetration of distributed energy resources in distribution networks is expected to bring operational challenges to network operators. It is commonly assumed that congestion management mechanisms are required to counteract the anticipated problems. However, to investigate such mechanisms, careful and detailed simulations are required to determine the exact impact and effectiveness in distribution networks. Simulations need to consider a detailed prosumer modelling, their participation in the energy markets, an accurate network representation and the effect of network tariffs, among others. Only then different congestion management mechanisms can be fairly assessed. This paper presents such a simulation platform as a first step towards a holistic assessment framework, and presents some exemplary case studies as a preliminary assessment.
\end{abstract}

Index Terms-Congestion management, Network tariffs, Distributed Energy Resources, Distribution network

\section{INTRODUCTION}

Many countries have set ambitious sustainability goals which require that power systems use primarily renewable generation sources, and that a large part of energy demand is electrified. For example, Denmark plans to reduce greenhouse gas emissions by $70 \%$ by 2030 (compared to the 1990 levels) and reach climate neutrality by 2050 [1], [2]. The electrification of demand will result in an increase of the number of installed electric vehicles (EVs) and heat pumps. Further, one of the means of replacement of fossil-fueled energy production is via residential photovoltaic (PV) systems, which are sometimes accompanied by battery storage systems. These distributed energy resources (DERs) challenge the operation of distribution networks (DNs), in the form of congestion and voltage deviations/range violations $[3] 1$ It is a rather popular opinion in the power systems literature that the high penetration of DERs will cause operational problems, and that eventually some sort of congestion management will be needed to resolve them, to avoid excessive grid investments [4].

However, it is not straightforward to assess if and how often the existence of large numbers of DERs will cause operational problems in the DN, and the answer is very case-specific. To investigate this, first, a proper case description must be

\footnotetext{
${ }^{1}$ Congestion refers to a situation where the power flowing through a component exceeds its physical and/or operational capabilities.
}

provided by specifying the network type/topology, the applied network tariffs and system prices, the inflexible consumption, the amount, type and placement of DERs, the objectives of the customers when controlling flexible demand and storage units, etc. Second, the result of the assessment should be provided in probabilistic terms because of the large number of involved uncertainties, such as electricity prices, weather conditions, EV driving patterns and the realization of inflexible demand, among others.

A number of studies that assess the impact of DERs on DNs exist in the literature, which typically apply and compare various congestion management mechanisms. The authors of [5] perform an assessment of various network tariff schemes in terms of predictability and cost-reflectivity, but without detailed modelling of customers, inclusion of prosumer/aggregator optimization or power flow simulation results. Instead, the effect of each house on network loading is estimated by using the Veelander load equation and Monte Carlo sampling, instead of conducting detailed simulations. The authors of [6] examine the effect of different tariff structures on prosumers based on cost minimization, but without consideration of the network. Ref. [7] conducts an assessment of the effect of widespread adoption of PV] and PV.battery systems. This is done by a bottom-up approach for residential customers, but the assessment is limited to the effect on system-level generation cost, rather than the DN-level.

Ref. [8] assesses the performance of various congestion management methods in terms of grid balance and value of flexibility on the balancing market. The work considers only EVs and it reveals that a peak tariff and a flexibility market can provide sufficient incentives to prevent grid congestion, but are not effective in the use of flexible demand for balancing under all circumstances. Ref. [9] analyzes cost-optimal operation of four different capacity-based grid tariff settings based on a Norwegian campus site with flexible demand. The study investigates how the frequency of adjusting the tariffs (weekly versus annually) influences the peak loads and cost savings. However, only EVs are considered in the analysis and the DN is neglected. The authors of [10] use an agent-based tariff simulator to assess the impact of different network tariff schemes on the consumption, production, and storage behavior of residential households. However, no network considerations are made and the assessment focuses on one congestion management mechanism. Further, only $[\mathrm{PV}]$ and storage systems 
are considered and the overall goal is to predict the impact of network pricing on the decision of customers to invest in such resources.

To the best of our knowledge, there is currently no complete assessment framework that considers all the necessary components to allow a realistic study of the impact of DERs and congestion management methods on DNs, under different conditions and inputs. The main contribution of this paper is to present a simulation platform that incorporates, among others, load modelling (DERs included), DN choice and power flows, consideration of different distribution system operator (DSO) and prosumer/aggregator strategies, and congestion management mechanisms. This platform forms the basis of a holistic assessment framework, which we are currently developing. The second contribution lies in using this platform to investigate the impact of DER placement and penetration levels, as well as different network pricing schemes, on DN loading and voltage levels. Further, we discuss those results with reference to modelling needs and limitations.

The remainder of the paper is structured as follows. Section II presents the developed simulation platform and all relevant components. In Section III a case study with real data is presented and various aspects of DER integration in DNs are assessed. Finally, Section IV concludes the paper and discusses the future direction of the presented work.

\section{Methodology}

In this section, we present the simulation platform which forms the core of the assessment framework for studying the impact of DERs and congestion management mechanisms on DNs The platform with its building blocks is shown in Fig. 1 and has three main parts: data input, simulation and assessment.

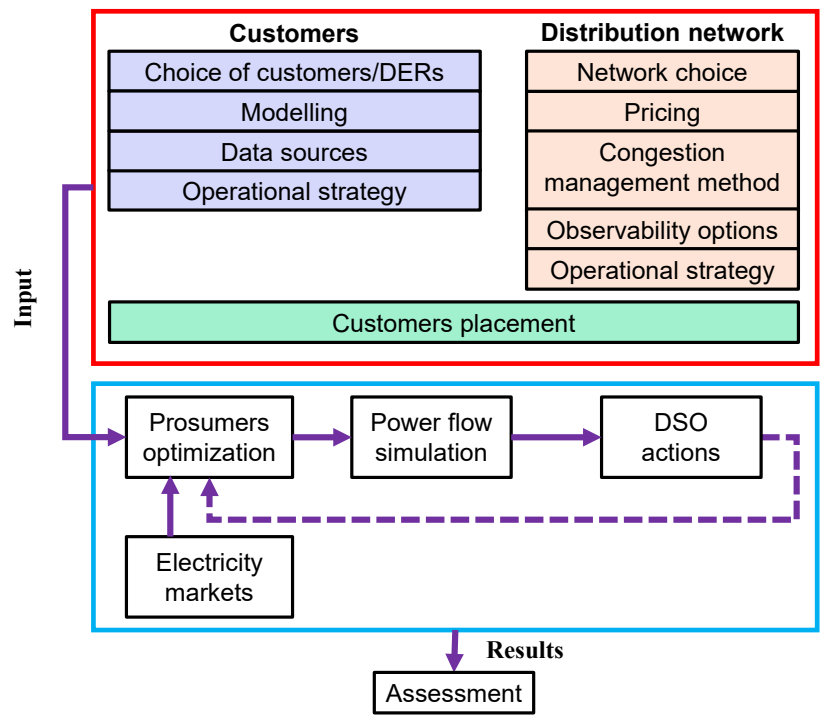

Fig. 1: Simulation platform with its constituent blocks

\section{A. Input}

The necessary input can be broadly divided into two categories: customers and network. We use the following input blocks for the customers category.
- Choice of customers: defining the overall level of inflexible consumption (low/medium/high), whether each customer owns a PV unit, an EV and a battery energy storage system (BESS), their parameters etc.

- Modelling: a modelling approach must be chosen to describe the operation of the aforementioned components (BESS, EV charging etc.).

- Data sources: the necessary inflexible consumption and EV driving/charging profiles, as well as $\mathrm{PV}$ production data must be collected.

- Operational strategy: this block defines the optimization problem of each customer, with the associated DERs. The objective could be the maximization of self-consumption or spot-price cost minimization, and the operational strategy incorporates all uncertainty considerations. Finally, the existence or absence of aggregators who control the flexible assets of the customers should be defined, together with the overarching aggregator optimization problem and goals, for example participation in the balancing market.

The following input blocks are defined for the network category.

- Network choice: here the DN to be studied is chosen.

- Pricing: it refers to the applied network pricing, i.e., flat or time-of-use tariffs, and transmission fees and taxes.

- Congestion management method: it defines the used method and its implementation, i.e., bilateral agreements or a local flexibility market.

- Observability option: this block defines the DSO s level of network observability, which is important in defining the operator's operational strategy.

- Operational strategy: this block defines how the DSO operates the network, i.e., if on-load tap changers are activated at specific voltage values, if protection is triggered at certain overloadings etc. This block can also define mandatory reactive power support, as is often the case with PV systems.

Finally, another important aspect of the assessment is the placement of the customers together with the DERs in the nodes of the chosen DN

\section{B. Simulation}

The following blocks are defined for this category.

- Prosumers optimization: this block solves the optimization problem of each prosumer (which may be part of an aggregator) and provides the active/reactive power setpoints.

- Electricity markets: this block provides the interaction with the electricity markets for allowing the prosumers/aggregators to participate in the wholesale/ancillary service markets.

- Power flow simulation: this block takes the aforementioned setpoints and runs the power flow in the DN

- DSO actions: depending on the observability of the $\mathrm{DN}$ the resulting power flows and the existence of a congestion management mechanism, the DSO may take actions, some of which may provide direct input to the prosumers, e.g., activating a flexibility service. 


\section{Assessment}

The assessment of the impact of DERs in DNs can be performed by using the obtained simulation data. Examples could be the calculation of overloadings of components and voltage levels, or the impact of different pricing schemes on aggregator costs and DSO revenue. Importantly, such a coherent framework allows a more systematic comparison of the chosen factors by using the same metrics.

\section{CASE STUdY}

\section{A. Simulation setup}

In our case study we use a semi-urban network model from [11], shown in Fig. 2 The topology of the DN is radial, the rated voltage is equal to $400 \mathrm{~V}$ and the transformer's capacity equal to $400 \mathrm{kVA}$.

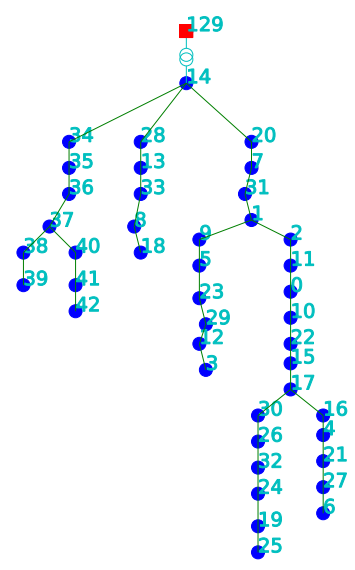

Fig. 2: Simulated network model

It should be noted that the referenced network contains only 40 individual customers. A single set of active and reactive power values is provided, which allows the user/investigator to obtain one snapshot of the power flows. These values are considerably higher than typical inflexible demand profiles. To obtain a realistic utilization of the network's transformer (equal to a maximum loading of $50 \%$ [12]), $4-6$ customers were assigned in each bus, leading to 200 loads. The residential inflexible load profiles are obtained by the Low carbon London project [13]. The yearly consumption ranges from 800 to $15000 \mathrm{kWh}$ per year (average value of $4000 \mathrm{kWh}$ ), with an average peak load of $3.5 \mathrm{~kW}$.

In this case study a $50 \%$ DER penetration ${ }^{2}$ is considered, with three scenarios: only EVs, only $\mathrm{PVs}$ and combinations of both types, referred to as EVPV. EV profiles are selected from [14], with a charger nominal capacity of $3.7 \mathrm{~kW}$ and a charging efficiency of $0.9 . \mathrm{PV}$ profiles are generated for the area of Copenhagen in year 2019 by using a simulator [15], with a power capacity of each PV] being equal to $5 \mathrm{~kW}$.

Spot prices for the DK2 area of Denmark and for year 2019 are downloaded from [16]. Both flat and time of use (ToU) tariffs are considered. In the former case a flat network tariff

\footnotetext{
${ }^{2}$ Penetration refers to the ratio of installed DERs to the total number of customers.
}

equal to $0.276 \mathrm{DKK} / \mathrm{kWh}$ is applied to each $\mathrm{kWh}$ imported by the customer. In the latter, a network tariff equal to 0.63 $\mathrm{DKK} / \mathrm{kWh}$ is applied between 17.00 and 20.00 from October to March; in all remaining hours it is equal to $0.236 \mathrm{DKK} / \mathrm{kWh}$ [17]. The rest of the price components are equal in both cases, with a tax of $0.9 \mathrm{DKK} / \mathrm{kWh}$ and a transmission tariff of 0.11 $\mathrm{DKK} / \mathrm{kWh}$. Retailer buy and sell prices are set equal to 1.25 and 0.75 times the spot prices, respectively. Finally, prosumers are subject to hourly net metering and receive the sell price when exporting energy at a given hour, or pay the buy price and the aforementioned taxes/tariffs, with a $25 \%$ VAT added. Time resolution is set to 1 hour for the whole simulated year and the load flow problem is solved via pandapower in Python 3 [18], and Mosek is used as an optimization solver [19].

\section{B. Impact of load placement}

Since the studied network is not accompanied by sufficient historical data and the time-series were retrieved as discussed in the previous subsection, first we investigate the effect of load placement. We start by placing a selected set of 200 customers only with inflexible load randomly in the nodes of the network, and run a power flow for the whole simulated year. We repeated the simulations with 50 different random load placements. The 5 th percentile of the 6 leaf bus voltages are shown in Fig. 3 (a). The effect of the exact load placement does not seem to have a substantial impact on bus voltages and the variability of the resulting percentiles is negligible.

We repeated the simulations by introducing $100 \mathrm{EVs}(50 \%$ EV penetration) and assume they are randomly assigned by customers, since system operator cannot change DER placement. EV charging is optimized to minimize prosumer cost (see the Appendix for more details). Even though voltage variability slightly increases (see Fig. 3 (b)), again the exact placement of the DERs does not have a significant impact on voltage.

\section{Assessment of network tariffs}

Next, we investigate the effect of employing a flat versus the specified $\mathrm{ToU}$ tariff for the $50 \% \mathrm{EV}$ scenario. Apart from the optimal EV charging, we consider the case where EVs charge as soon as they plug-in, and charge until their energy needs are covered; we call this case normal charging.

Table I presents the annual cost for the EV demand of prosumers and the associated DSO revenue. We present only the EV-related consumer cost andDSO revenue because inflexible demand is insensitive to the tariffs, and including it does not facilitate the interpretation of the results. With flat tariffs, DSO revenue remains unaffected by optimal charging, since the consumed energy remains the same. Optimal charging reduces costs by $3.0 \%$, since the average daily $\mathrm{EV}$ demand is relatively small $(7.5 \mathrm{kWh})$, and because energy costs are approximately equal to only $20 \%$ of the final electricity price.

The introduction of ToU tariffs does not result in a noticeable change in either charging costs or DSO revenue under normal charging. However, a ToUltariff with optimal charging reduces DSO revenue by shifting a part of the EV load away from hours with higher tariffs; a similar effect is observed on the charging cost, which drop by a larger amount compared to 
(a) Inflexible load placement

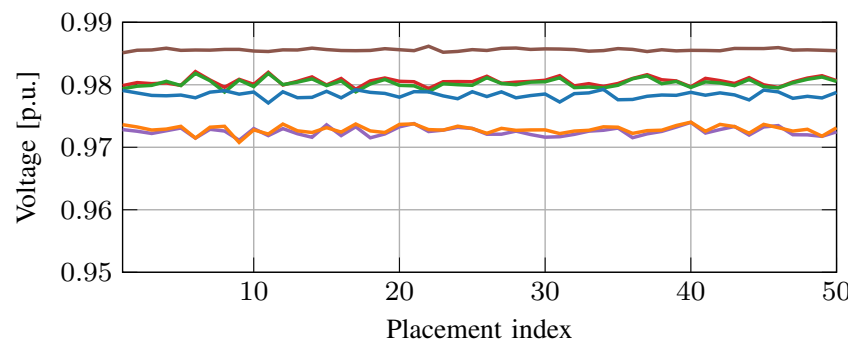

(b) $50 \%$ EV placement

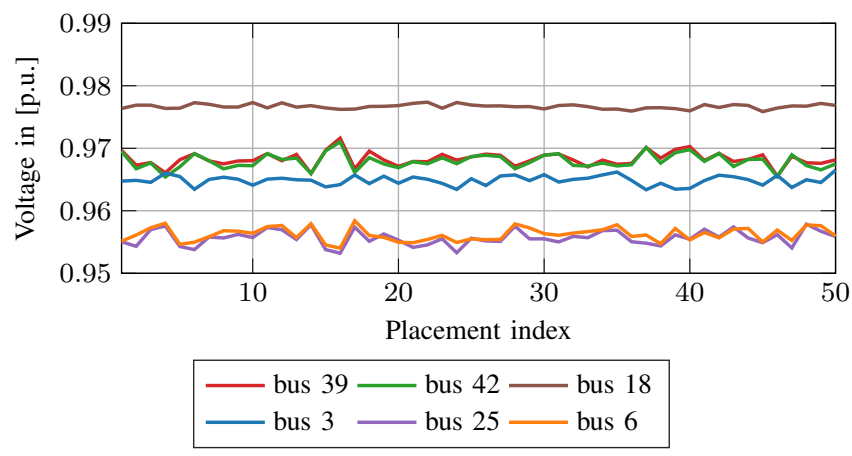

Fig. 3: 5th percentile of bus voltage for each random placement

the flat tariff due to the reduced DSO revenue. Notice that the additional charging cost reduction is equal to the DSO revenue loss due to optimal charging.

\begin{tabular}{ccc} 
& Normal charging & Optimal charging \\
\hline \hline \multicolumn{3}{c}{ Flat Tariff } \\
\hline EV charging cost & 707.56 & 686.24 \\
DSO revenue & 94.22 & 94.22 \\
\hline & ToU Tariff \\
\hline EV charging cost & 707.28 & 673.57 \\
DSO revenue & 93.99 & 84.08 \\
\hline
\end{tabular}

TABLE I: Annual EV charging cost and DSO revenue in thousands DKK for a $50 \% \mathrm{EV}$ penetration scenario.

The asset utilization under the two tariff schemes with optimal charging are shown in Tab. II] where the 95-th and $99-$ th percentiles are reported. A $50 \% \mathrm{EV}$ penetration does not lead to transformer congestion, whereas congestion appears $5 \%$ of the time in line-24 (the line between node 14 and 20). What is interesting to note is that both tariff schemes lead to the same metrics values for asset utilization. This happens because under optimized charging, EVs mostly charge at early morning hours when system prices are lowest. Therefore, a high network tariff between 17.00 and 20.00 does not affect the charging profiles in most cases, since system prices are typically high during those hours. As a result, the particular ToUtariff choice does not contribute to the reduction of congestion in the considered case, where a significant part of the total network load comes from EV charging needs.

\begin{tabular}{ccc} 
& ToU Tariff & Flat Tariff \\
\hline \hline & Transformer 400kVA \\
\hline 99th percentile & $90.7 \%$ & $90.7 \%$ \\
95th percentile & $67.9 \%$ & $67.9 \%$ \\
\hline \multicolumn{3}{c}{ Line-24 0.27kA } \\
\hline 99th percentile & $129.4 \%$ & $129.4 \%$ \\
95th percentile & $99.7 \%$ & $99.7 \%$ \\
\hline
\end{tabular}

TABLE II: Asset utilization with optimal charging for a $50 \%$ EV penetration scenario.

(a) Voltage for different $\mathrm{EV}$ penetration levels

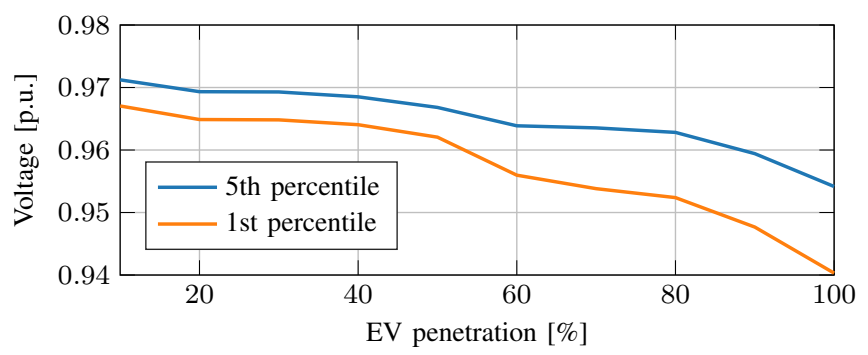

(b) Voltage for different $\mathrm{PV}$ penetration levels

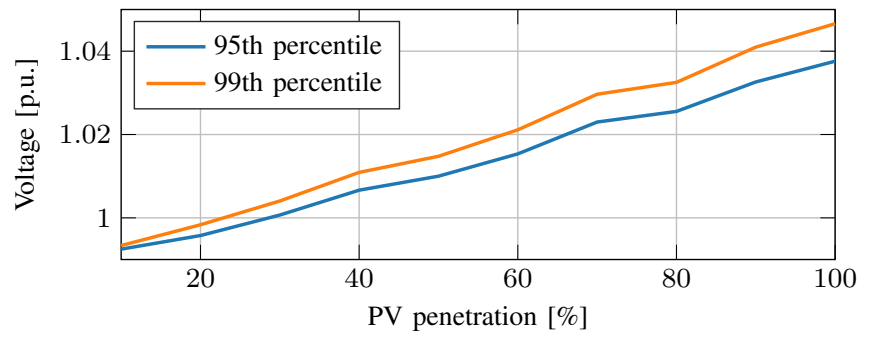

Fig. 4: Voltage variation under different DER penetration levels at bus 25 .

\section{Investigation of DER penetration levels}

Finally, we explore the impact of DER type and penetration on network voltage. Voltage at bus 25 is selected in the examined network because it is the bus that has the lowest voltage values. Fig. 4 (a) shows that EV penetration does not result in significant voltage drops even with a share of $50 \%$. However, after this level the reduction in voltage becomes steeper, leading in values below 0.94 p.u. $1 \%$ of the time. In contrast to that behavior, an increasing $\mathrm{PV}$ penetration increases voltage linearly, with the maximum value still lying within the safety limits. Tab. III compares the utilization of the transformer and line 20 for three scenarios: no DER, $50 \%$ EV and 50\% EVPV under a flat tariff. The introduction of EVs results in a significant increase in loading, which leads to frequent overloadings in line 24, whereas the transformer is above $90 \%$ of its rated capacity for approximately 90 hours in the given year. The existence of PV] systems slightly reduces network loading via self-consumption. This reduction is relatively small because typically overloadings occur at early morning hours with low energy prices and zero $\mathrm{PV}$ production. 


\begin{tabular}{cccc}
\hline & No DERs & $50 \%$ EV & 50\% EVPV \\
\hline \hline \multicolumn{3}{c}{ Transformer 400kVA } \\
\hline 99\% percentile & $49.6 \%$ & $90.7 \%$ & $85.3 \%$ \\
$95 \%$ percentile & $43.5 \%$ & $67.9 \%$ & $65.8 \%$ \\
Average loading & $26.1 \%$ & $37.4 \%$ & $32.4 \%$ \\
\hline \multicolumn{4}{c}{ Line-24 0.27kA } \\
\hline 99\% percentile & $70.2 \%$ & $129.4 \%$ & $121.5 \%$ \\
95\% percentile & $61.5 \%$ & $99.7 \%$ & $93.4 \%$ \\
Average loading & $36.7 \%$ & $53.0 \%$ & $45.8 \%$ \\
\hline
\end{tabular}

TABLE III: Asset utilization for the no DERs case vs the $50 \%$ DER penetration scenarios.

\section{DiscusSiON AND CONCLUSION}

This paper makes a step towards developing a holistic framework to assess the impact of DERs and the effectiveness of congestion management(tariff) methods in an unified way, by developing a Python-based simulation platform. The challenge with such assessments lies in the fact that a wide variety of factors and options affect the results, making it hard to derive concrete and sound conclusions. Since a few critical assumptions or simplifications may lead to very different results, the use of a systematic analysis framework is fundamental to achieve coherent results.

A case study was used to exemplify how the impact of a few factors can be assessed. It was shown that the introduction of ToU tariffs does not prevent overloadings under optimal EV] charging, since those usually occur in hours outside high tariff period. However, such ToU tariffs are usually placed to counteract the impact of normal charging, which usually leads to high evening peaks. Under optimal charging it is very likely that operators would design those tariffs in a different manner. It was also shown that the exact load placement does not seem to play a particular role in network voltage, but a more thorough investigation is needed with regards to line currents. The common assumption of $50 \%$ max transformer loading can be met with different load placements along the network's branches, so a more careful assessment is required to determine the effect of placement on line loading. In our future work we will expand the presented simulation platform to a holistic detailed DER and congestion management methods assessment framework.

\section{APPENDIX}

Inflexible consumption is denoted by $p_{t}^{1}$ and $\mathrm{PV}$ production by $p_{t}^{\mathrm{g}}$ at each step $t$. Let $p_{t}^{\mathrm{ev}}$ as EV charging, $p_{t}^{\mathrm{b}}$ and $p_{t}^{\mathrm{s}}$ be the energy bought from or sold to the retailer, at corresponding prices $\lambda_{t}^{\mathrm{b}}$ and $\lambda_{t}^{\mathrm{s}}$. The prosumer's cost minimization problem is formulated as

$$
\begin{gathered}
\min _{\mathcal{D}} \quad \sum_{t \in \mathcal{T}}\left(p_{t}^{\mathrm{b}}\left(\lambda_{t}^{\mathrm{b}}+c_{t}\right)-p_{t}^{\mathrm{s}} \lambda_{t}^{\mathrm{s}}\right) \\
\text { s.t. } \quad p_{t}^{\mathrm{b}} \geq 0, \quad p_{t}^{\mathrm{s}} \geq 0, \\
p_{t}^{\mathrm{b}}-p_{t}^{\mathrm{s}}=p_{t}^{\mathrm{ev}}+p_{t}^{\mathrm{l}}-p_{t}^{\mathrm{g}},
\end{gathered}
$$

Note that only energy imports $p_{t}^{\mathrm{b}}$ are subject to tariffs/taxes $\left(c_{t}\right)$. Each EV undergoes $q \in \mathcal{Q}$ sessions in a given day. In each session: plug-in time $t_{q}^{\text {in }}$, plug-out time $t_{q}^{\text {out }}$ and charging needs $d_{q}$ in $\mathrm{kWh}$. We define $\mathcal{T}^{\mathcal{D}}$ as the ordered set of the union of the time steps where the EV is plugged-in, i.e., $\left[\left[t_{1}^{\text {in }}, t_{1}^{\text {out }}\right] \cup\left[t_{2}^{\text {in }}, t_{2}^{\text {out }}\right], \ldots\right]$. Let $X_{t}$ be the $\mathrm{EV}$ state-of-charge expressed in $\mathrm{kWh}, \hat{p}^{\mathrm{ev}}$ the nominal charging power, $\eta$ the charging efficiency, $C$ the battery capacity and $C^{\text {set }}$ the stateof-charge at the end of each session. The EV constraints apply:

$$
\begin{array}{lc}
0 \leq p_{t}^{\mathrm{ev}} \leq \hat{p}^{\mathrm{ev}}, \quad 0.1 C \leq X_{t} \leq C & \forall t \in \mathcal{T}^{\mathcal{D}} \\
\sum_{t=t_{q}^{\text {in }}}^{t_{q}^{\text {out }}} p_{t}^{\mathrm{ev}} \eta=d_{q}, & \forall q \in \mathcal{Q} \\
X_{t}=X_{t-1}+\eta p_{t}^{\mathrm{ev}}, & \forall t \in \mathcal{T}^{\mathcal{D}} \\
X_{t_{q}^{\text {in }}-1}=C^{\text {set }}-d_{q}, \quad X_{t_{q}^{\text {out }}}=C^{\text {set }}, & \forall q \in \mathcal{Q}
\end{array}
$$

\section{REFERENCES}

[1] The Danish Government, "The danish government's long-term strategy for global climate action," 2020.

[2] J. Østergaard, C. Ziras, H. W. Bindner, J. Kazempour, M. Marinelli, P. Markussen, S. H. Rosted, and J. S. Christensen, "Energy security through demand-side flexibility: The case of denmark," IEEE Power and Energy Magazine, vol. 19, no. 2, pp. 46-55, 2021.

[3] A. Pillay, P. Karthikeyan, and D. P. Kothari, "Congestion management in power systems - a review," International Journal of Electrical Power \& Energy Systems, vol. 70, pp. 83-90, 2015.

[4] S. Huang, Q. Wu, Z. Liu, and A. H. Nielsen, "Review of congestion management methods for distribution networks with high penetration of distributed energy resources," Proceedings of 2014 ISGT Europe, 2014.

[5] M. Nijhuis, M. Gibescu, and J. F. Cobben, "Analysis of reflectivity $\&$ predictability of electricity network tariff structures for household consumers," Energy Policy, vol. 109, no. July, pp. 631-641, 2017.

[6] S. Bjarghov, M. Korpas, and S. Zaferanlouei, "Value comparison of EV and house batteries at end-user level under different grid tariffs," 2018 IEEE International Energy Conference, ENERGYCON 2018, pp. 1-6, 2018.

[7] D. Jaglal, A. T. Procopiou, K. Petrou, and L. F. Ochoa, "Bottomup modeling of residential batteries and their effect on system-level generation cost," Electric Power Systems Research, vol. 189, no. April, p. 106711, 2020.

[8] A. Stawska, N. Romero, M. D. Weerdt, and R. Verzijlbergh, "Demand response: For congestion management or for grid balancing?," Energy Policy, vol. 148, p. Part A, 2021.

[9] S. Backe, G. Kara, and A. Tomasgard, "Comparing individual and coordinated demand response with dynamic and static power grid tariffs," Energy, vol. 201, 2020.

[10] M. Manuel de Villena, J. Jacqmin, R. Fonteneau, A. Gautier, and D. Ernst, "Network tariffs and the integration of prosumers: The case of wallonia," Energy Policy, vol. 150, p. 112065, 2021.

[11] S. Meinecke, D. Sarajlić, S. R. Drauz, A. Klettke, L.-P. Lauven, C. Rehtanz, A. Moser, and M. Braun, "Simbench-a benchmark dataset of electric power systems to compare innovative solutions based on power flow analysis," Energies, vol. 13, no. 12, 2020.

[12] L. González-Sotres, C. Mateo Domingo, Sánchez-Miralles, and M. Alvar Miró, "Large-scale mv/lv transformer substation planning considering network costs and flexible area decomposition," IEEE Transactions on Power Delivery, vol. 28, no. 4, pp. 2245-2253, 2013.

[13] U. P. Network, "Low carbon london project." https://innovation. ukpowernetworks.co.uk/projects/low-carbon-london/

[14] D. for Transport, "Electric chargepoint analysis 2017: Domestics," 2018.

[15] S. Pfenninger and I. Staffell, "Long-term patterns of european pv output using 30 years of validated hourly reanalysis and satellite data," Energy, vol. 114, pp. 1251-1265, 2016.

[16] E. D. SERVICE, "Elspot prices." https://www.energidataservice.dk/ tso-electricity/elspotprices/

[17] D. D. Radius, "Radius tariff scheme." https://radiuselnet.dk/elkunder/ priser-og-vilkaar/tariffer-og-netabonnement/

[18] L. Thurner, A. Scheidler, F. Schafer, J. Menke, J. Dollichon, F. Meier, S. Meinecke, and M. Braun, "pandapower - an open source python tool for convenient modeling, analysis and optimization of electric power systems," IEEE Transactions on Power Systems, vol. 33, no. 6, pp. 65106521, 2018.

[19] M. Aps, "Mosek optimization suite," 2018. 